\title{
«Das Leiden Christi?! Aus Papier?!»
}

\author{
Über Holz/Schlafs Die papierne Passion (1890)
}

Andreas Mauz

\section{I.}

Die Passion, die im Titel von Arno Holz und Johannes Schlaf gemeinsam verfasster dramatischer Skizze angekündigt wird, lässt auf sich warten. Erst im letzten Viertel kommt sie ins Spiel, eine aus Zeitungspapier gebildete Passionsszene: «Sehn Se, meine Herrschaften! Det is det Kreiz unsers Herrn Jesus Christus! Det Kreiz, an den er jahangen hat, un an den se ihn den Essigschwamm reichten. [...] Det hier is Joljatha, jenannt de Schädelstätte! Se wissen doch!» (30) Was der Einführung der Passion voraus geht, ist mit dem ursprünglichen Untertitel des Textes - Eine Berliner Studie - gut beschrieben. ${ }^{1}$ Schauplatz der Handlung ist "[e]ine kleine berliner Küche, [...] um die Weihnachtszeit» (7), das Personal ist stark typisiert. Da ist Mutter Abendroth'n, die Kartoffeln reibende Wirtin, ihre Pflegetochter Wally, «ein kleines, blondes, vermeckertes Ding von elf Jahren» (8), der sensible Student Haase, sein weltgewandtes Pendant, der Bohemien Röder, und schliesslich Olle Kopelke, der rheumatische Hausfreund, ehedem an Mutter Abendroth'n interessiert, heute mehr an ihren Kartoffelpuffern. Zwischen ihnen entfaltet sich auf einer Länge von 28 Seiten ein Geschehen, das zu denken gibt.

In der germanistischen Forschung hat der Text eher wenig Aufmerksamkeit gefunden - deutlich weniger zumindest als die Prosaszene Papa Hamlet (1889) und das Kurzdrama Die Familie Selicke (1890), die ihn im Zweitdruck, den «Studien» (3; Vorwort) der Neuen Gleise, begleiten. ${ }^{2}$ Sie wurden Gegenstand zahlreicher Untersuchungen und gehören zum Regelbestand von Anthologien

\footnotetext{
1 Arno Holz/Johannes Schlaf, Die papierne Passion. (Olle Kopelke.). Eine Berliner Studie, in: Freie Bühne für modernes Leben, Jg. 1, H. 9 (2.4.1890), 274-288. Zitate werden nach dem Zweitdruck im Haupttext nachgewiesen: Arno Holz/Johannes Schlaf, Neue Gleise. Gemeinsames von Arno Holz und Johannes Schlaf. In drei Teilen und einem Band, Berlin: Fontane 1892, 7-35.

2 Die eingehendste Kommentierung bietet meines Wissens: R. Whitinger, Johannes Schlaf and German Naturalist Drama, Columbia, SC, 1997, 38-45.
} 
des Naturalismus. Dass die Papierne Passion vergleichsweise wenig beachtet blieb, mag erstaunen. Immerhin eröffnet sie die Neuen Gleise und fungiert auch als Überschrift ihres ersten Teils (die beiden anderen sind nach den erwähnten klassischen Beiträgen benannt). Erstaunlich ist aber auch, dass der Text im Umfeld theologischer Literaturrezeption keine Beachtung fand. Denn gerade in seiner näher zu bestimmenden - Theologielosigkeit ist er auch von theologischem Interesse. Vor diesem Hintergrund liegt der Akzent im Folgenden ganz auf dem Text selbst. Er wird immanent bearbeitet, wobei die Lektüre am Leitfaden der Titelvokabel der «Passion» erfolgt. Nicht Rechnung getragen wird demnach den vielfältigen Verbindungen, die vom Text zum Ganzen der Neuen Gleise ausgehen (etwa ist auch die Familie Selicke ein Weihnachtsstück) bzw. den poetologischen Zusammenhängen, in die er einzuzeichnen wäre (bzw. sich selbst in programmatischer Weise einzeichnet, wie bereits im Titel anklingt). ${ }^{3}$ Nebst der gesamten Bewegung des Erzählten, des Stoffs, muss zunächst aber dennoch auch die Eigenart des Erzählens, derVermittlung des Stoffs, skizziert werden.

II.

Was sofort ins Auge springt, ist die raum-zeitliche Geschlossenheit der szenischen Repräsentation. Der Schauplatz ist ein einziger, Mutter Abendroth'ns Küche, und die Darstellung folgt nicht nur strikt der Chronologie - was gezeigt wird, ist, ohne jegliche Unterbrechung, ein einziges Gespräch. Würde der Text inszeniert, die reale Spielzeit und die fiktive gespielte Zeit deckten sich genau.Andere Zeitebenen und Schauplätze werden evoziert (letztere insbesondere durch ihre akustische Vergegenwärtigung; 15, 32), nicht aber gezeigt.

Wie bei Dramen üblich, besteht das Textsubstrat aus einem bereits typographisch unterscheidbaren Haupttext (den Figurenreden) und einem Nebentext (den Regieanweisungen, die Orte, Zeiten, Figurencharakteristik, Tonalität, nichtverbale Handlungen etc. fest halten). Holz/Schlaf realisieren diese Anlage aber insofern in eigener Weise als die petit gesetzten Nebentexte deutlich mehr sind als blosse

3 Zu diesen Zusammenhängen: F. Gelzer, «Ein einziges grosses Experiment». Zu Arno Holz' und Johannes Schlafs «Neue Gleise` (1892), erscheint demnächst in: Sprachkunst. Beiträge zur Literaturwissenschaft 39/1 (2008). Den Hinweis auf die Papierne Passion verdanke ich Florian Gelzer (Bern), der auch für eine hilfreiche Kommentierung dieses Beitrags zur Verfügung stand. Für den gleichen Dienst auch mein Dank an Stefan Berg (Zürich). 
Regieanweisungen. Bereits aufgrund ihres Umfangs - und trotz des kleineren Schriftgrads - wirken sie nicht sehr «neben`; und zählte man die Zeichen beider Korpora aus, so dominierten sie vermutlich. Inhaltlich bieten sie darüber hinaus einen Grad an Präzision, wie er im Blick auf eine Dramatisierung weder zu leisten, noch gängig ist:

\begin{abstract}
«Auf dem zertrampelten Schnee liegen breite, gelbe Lichtstreifen von den hellen Hoffenstern und die rothen, unstäten Reflexe der grossen Laterne. Das Stampfen und Dröhnen und Quietschen der Maschinen und, von den Hinterhäusern her, noch immer, melodisch, das Tenorhorn ...» (32)
\end{abstract}

Die Nebentexte referieren aber nicht nur akustische Signale, die von Aussen in die Küchenszene dringen, sie bieten auch Originaltöne, Stimmen im Hof des Mietshauses. Von ihnen abgesehen geht die ¿Zweidimensionalität〉 des Textraums einher mit einer strikten linguistischen Differenz: In den erweiterten Regieanweisungen spricht man Hochdeutsch und nicht, je nach Figur, berlinerisch. Die akribisch genaue Repräsentation gesprochener Sprache in der Schrift zeigt sich aber nicht nur im Dialektalen. Auch die offensive Nutzung der Interpunktion (etwa zur Gestaltung von «Crescendo)-Effekten bzw. der Abbildung der Intonation: «Hil-fe! Hil-feee!! [...] Hiiil-fe!!! [sic]», 30) oder die Integration von Onomatopoetica («M!», 8; «Pff!», 11) zielen auf eine konsequent «naturalistische〉 Darstellung. Man hat es, kurz gesagt, mit einer Mischgattung zu tun, einem Lesedrama, das dem uobjektiven〉 dramatischen Modus eine ähnlich intensive narrative Stimme an die Seite stellt; aber auch sie hält sich an das empirisch Wahrnehmbare und verzichtet etwa vollständig auf eine psychologisierende Innenschau oder andere Formen der deutenden Distanzierung vom Geschehen.

Die dergestalt repräsentierte Handlung setzt ein mit Mutter Abendroth'n, die allein in der Küche sitzt und Kartoffeln reibt. Bald darauf tritt Wally ein, mit zweistündiger Verspätung, was ihre Pflegemutter zu wüsten Beschimpfungen veranlasst. Wally, die «dusslichte Droomlade» (9), flieht wieder hinaus. Bald darauf erscheint sie erneut, diesmal begleitet vom nervös rauchenden Herrn Haase. Sie sprechen vom schlechten Wetter bis Haase schliesslich sein Anliegen vorbringt: Geduld mit der Miete, "[n]ur bis zum Fünfzehnten!» (13) Er bekommt den Aufschub und eine Tasse Kaffee. Dann wird deutlich, weshalb Mutter Abendroth'n so schlecht auf Wally zu sprechen ist. Sie ist nicht nur die uneheliche Tochter ihrer Schwester - ihr eigenes Kind, das geliebte «Marieken» (14) ist mit 12 Jahren gestorben: «Weine doch nicht, liebes Mutterchen! Mir ist ja wohl! 
Ich komme ja nun zum lieben Gott! Da sehn wir uns alle wieder!» Beeindruckt davon, "[w]at det Kind schon fier 'ne Sprache hatte» (15), fällt ihre Mutter, zitierend, für einmal ins Hochdeutsche. Die gedrückte Stimmung - «Wally hat sich gegen das Fenster gedrückt, sie schüttelt sich vor Frost» (16) - hebt sich als Herr Röder eintritt, nach dem Abendessen fragt und mit Mutter Abendroth'n zu schäkern beginnt. Kaum hat sich Röder verabschiedet, folgt der letzte Gast, Olle Kopelke. Er wird von Wally begeistert begrüsst, und während der ganzen Szene hängt sie wenn immer möglich an ihm. Kopelke, Frohnatur mit «gutmüthigen Augen» und «glattrasirte[m] dicke[m] Gesicht» (20), bekommt wie üblich seine «rohe[n] Bollen» (21), Zwiebeln, die er zur Regulierung seines Verdauungsapparats benötigt. Das Gespräch dreht sich um Allgemeinplätze, Wally wird von Zeit zu Zeit ausgeschimpft, und Kopelke ebenso wie Haase verfolgen aufmerksam die allmähliche Verfertigung der Kartoffelpuffer. Und schliesslich liefert Haase das Stichwort, das seinerseits die Passionsthematik auf den Plan ruft.

Auf Kopelkes Frage, ob es nicht eine "scheene Zeit is [...], det Studier'n», antwortet er: "O ja! Aber manchmal etwas roh! Etwas sehr roh!» Diese Antwort weckt in Kopelke eine Erinnerung: «[R]oh, meen' Se? Ja, da hab'n Se eejentlich nich so janz Unrecht! Und da weer'ck Ihn'n ooch mal 'ne scheene Jeschichte erzeehl'n.» (26f) Die Erzählung wird allerdings aufgeschoben, da die Erinnerung an sie auch diejenige an eine andere alte Geschichte weckt: eben jene, dass Mutter Abendroth'n und Kopelke sich früher einmal näher standen, dass sie, wäre der reiche Fritze nicht gewesen, vielleicht geheiratet hätten. Mutter Abendroth'ns Versuch, ihm durch Kartoffelpuffer den Mund zu stopfen, gelingt. Und der Kartoffelpuffer animiert Kopelke zu einem Reim, der ihn wieder auf die Spur der eigentlich zu erzählenden Geschichte bringt, nun erstmals das semantische Feld der Passion betretend: «Jesus sprach zu seine Jinger: / Wer keen Leffel hat, isst mit de Finger! / So! [...] / Er knippst sich die Zuckerkrümelchen ab.» (28)

Die Geschichte ist folgende: Kopelke hat in Greifswald Recht studiert und schnitt Silhouetten, um sich etwas dazu zu verdienen. "Und da hätt' ick beinah' mal in'ne Kneipe von de Theologen eklige Keile jekriegt, indem det ick det Leiden Christi ausschnitt!» (28) Ob die «Jeschichte», die ja eine «scheene» sein soll, damit bereits zu Ende ist, bleibt unklar.Vielleicht wird Kopelke auch nur an der Fortsetzung ihrer Erzählung gehindert, denn «Wally ist sehr interessirt auf Olle Kopelke zugehüpfelt»: «Au ja, Olle Kopelke kann det Leiden Kristi ausschneiden, det janze Leiden Kristi! [...] Du! Schneid' doch mal 
det Leiden Kristi aus! Ja? Mach’» (28) Wallys Enthusiasmus führt dazu, dass die Erzählung der Geschichte übergeht in ihre teilhafte Reinszenierung: Die papierne Passion, die beinahe zum Anlass einer Prügelei wurde, wird erneut gebaut. Wally sorgt für Papier, und da kein Briefbogen zur Hand ist, reicht sie Kopelke den «Localanzeiger»: «S. Maj. der Kaiser haben geruht ... hm! [...] Na, un hier? [...] In't Closette gestochen! [...] Wat nich so alles in de Welt passiert!» (29) Als erstes legt Kopelke einen grossen Papierschnitzel, «der wie ein Kreuz aussieht», auf den Tisch, er «hustet [...] und räuspert sich ein paar Mal pathetisch: «Sehn Se, meine Herrschaften! Det is det Kreiz unsers Herrn Jesus Christus!» Wallys Hinweis, dass «jop janich mal 'n Herr Kristus dran" (30) sei, übergeht er und bastelt aus zwei weiteren Schnitzeln Golgatha.

Dieses Bastelidyll - Mutter Abendroth'n sieht Kopelke über die Schultern zu, sie "hat sich einen Puffer zusammengerollt und kaut behaglich" (30) - wird unvermittelt von Aussen aufgebrochen: Aus dem Hof sind dumpfe Schläge zu hören, und eine Frau schreit grell um Hilfe. Der Schlosser, der im Parterre wohnt, ist ein Trinker und verprügelt seine Frau. Mutter Abendroth'n, Wally und Herr Haase, am ganzen Körper zitternd, schauen aus dem Fenster. Im Hof herrscht Aufruhr, es wird nach dem Schutzmann gerufen, man soll die Türe einschlagen. Durch das geöffnete Fenster zieht ein Windstoss in die Küche und verwirbelt die Zeitungsfetzen. Haase, noch immer zitternd, versucht mühsam sie aufzufangen: «Um Gottes-wil-len...» (32) Schliesslich kommt ein Schutzmann, die Tür wird aufgebrochen, die Lage beruhigt sich. Mutter Abendroth'n lehnt sich noch immer zum Fenster hinaus und spricht mit jemand, obwohl Kopelke sie bittet, es doch endlich zu schliessen. Sie weiss dann zu berichten, dass der Schlosser mit einem Beil auf seine Frau - schwanger - losgegangen sei, das Blut sei ihr nur so vom Kopf herunter gelaufen. Mutter Abendroth'n ist ausser sich und zetert, bis Kopelke, der längst wieder am Tisch sitzt und das Kreuz an seinen Ort gelegt hat, meint: «Na, rej’ Dir man wieder ab, Mutter!» (33) Das erinnert sie daran, dass Herr Röder sein Abendessen noch nicht bekommen hat. - Auch in der Küche kehrt wieder Ruhe ein, die Arbeit an der Passion geht weiter:

«[...] Na, nu passen Se mal uff, werther junger Herr! - Det wah also det Kreiz un det hier der Berg Joljatha! Hier, diese beeden Schnitzelkens sind die beeden Schechers! Zu den Een'n sagt der Herr Christus: Wahrlich, ick sage Dir, heite noch wirst Du mit mir in't Paradiese sind!» (34) 
Kaum ist die ganze Szenerie aufgebaut - Kriegsknechte, Mantel, Würfel, Maria, Johannes, Stock mit Schwamm, Emmaus-Jünger -, wird sie zerschlagen. Wally hat sich dem Tisch genähert und mitten in die Schnitzel gepustet: «Sie fliegen über den ganzen Tisch hin auseinander. [...] Nur das Kreuz ist schief auf dem Tische liegen geblieben.» (34) Kopelke nennt Wally «unjezogen», sie aber lacht nur, worauf er sich aus dem Kreuz einen «Fidibus»«kneift», einen Anzünder für seine Zigarre; «Herr Haase dreht stumpfsinnig die beiden Jünger von Emmaus zwischen den Fingern.» (35) Das Ende der papiernen Passion geht über ins Ende der dramatischen Szene selbst: Kopelke kommt kurz noch einmal auf seinen ursprünglichen Erzählanlass zu sprechen - «Is det nich sonderbar? Schliesslich kann Eener aus so wat 'n Spielzeich machen! [...] Dabei hätt'n se mir doch mal beinah eklig drum verhau'n! [...] Ween eener so nimmt: schliesslich is det doch 'ne putzige Welt!» (35) - und bittet Wally (und das ist der Schlusssatz), ihm noch einen Puffer zu reichen.

III.

Vor diesem Hintergrund kann nun weiter gefragt werden: Was heisst hier «Passion»? Aus der Sicht der Figuren bezeichnet der Begriff (vom Titel abgesehen erscheint er nur zwei Mal ausdrücklich; 31,34) die Summe der Personen und Gegenstände, die Kopelke aus Papier formt. Und tatsächlich ist es gleichsam das Inventar (Figuren, Gegenstände, Schauplätze) der Passionsgeschichte, welches ins Spiel kommt, nicht aber die Geschichte selbst, die Ereignisse, die sich mit einer Figur oder einem Gegenstand verbinden. Kopelke meint, sich diesbezüglich auf ein funktionierendes kulturelles Gedächtnis verlassen zu können: «Se wissen doch!» (30) Die blosse Erwähnung etwa der Mutter und des Jüngers, den er lieb hatte (34), soll die Szene ihrer Familiarisierung durch den johanneischen Jesus evozieren (Joh 19,25ff). Dass eine Figur ausdrücklich mit einem Handlungselement verbunden wird - «Zu den Een'n [der Schächer] sagt der Herr Christus: Wahrlich, ick sage Dir [...]» (34) -, ist die Ausnahme.

Gerade die Einspielung von Jesus-Zitaten (Zitaten des Jesus der Evangelien) macht aufmerksam auf eine signifikante Absenz. Wally hat nämlich recht: Die papierne Passion weist ein Kreuz auf, aber an ihm ist «janich mal 'n Kristus dran» (30). Ob eine Passion ohne Schmerzensmann Passion genannt zu werden verdient, sei dahin gestellt; seine Absenz weckt in jedem Fall einen Interpretationsbedarf - auch im Sinne der schlichten Rückfrage, wo der Leidende denn 
sein könnte, wenn er nicht da ist, wo er 〈hin gehört). - Was sich anböte, wäre die nahe liegende Antwort, die Passion werde eben überblendet mit der ihr folgenden narrativen bzw. heilsgeschichtlichen Sequenz der Auferweckung. Der Korpus fehlt, weil Jesus auferweckt wurde. Diese Lesart würfe, von ihrer allzu schnellen Erledigung der Irritation durch einen theologischen Reflex abgesehen, aber die Frage auf, wie es sich denn mit den Emmaus-Jüngern verhält. Auch sie (die an sich gar nicht in die Passionsszene gehören), sind - bevor sie zerknürgelt werden - allein unterwegs; dies zumindest ist aufgrund der ausdrücklichen Informationen anzunehmen. Und man wird auch keine technischen Gründe für die Absenz in Anschlag bringen können; Kopelke versteht sich grundsätzlich ja durchaus auf die Herstellung menschlicher Silhouetten. - Es ist ein Kreuz ohne Kreuzigung, ohne Gekreuzigten.

Das leere Kreuz gibt Anlass, den Horizont zu öffnen, die Szene der papiernen Passion also nicht nur für sich zu betrachten, sondern als Szene in der Szene. In dieser weiteren Perspektive stellt sich die Absenz des Leidenden möglicherweise anders dar. Und tatsächlich stösst man, so gesehen, alsbald nicht auf den, wohl aber auf die Leidenden, im Feminin Plural. Nur wenige Zeilen nach Wallys Hinweis auf die Absenz Jesu kommt die geschundene Frau des Schlossers ins Spiel, ihr Haupt voll Blut und Wunden. Aber auch Wally selbst, welche die Reinszenierung der Passion veranlasst, ist erkennbar als Jesusfiguration: «Sie drückt die Stirn gegen das Holzkreuz» (26), und sie trägt ihr Kreuz als ungeliebtes Kind einer Pflegemutter (in ihr permanent die Erinnerung an den Verlust des eigenen Kindes wach haltend) und eines doppelt abwesenden Vaters, des leiblichen ebenso wie des Pflegevaters.

Diese Zuordnungen sind als solche banal. Wer so interpretiert, bewegt sich in einem Schema, das im Umkreis theologischer Literaturrezeption nur zu beliebt ist. Darüber hinaus gehendes lässt sich fest halten, wenn die Beobachtungen noch einmal an die Perspektive der Figuren zurück gebunden werden. So betrachtet zeigt sich eine geradezu gespenstische Isolation der papiernen Passion. Dass die reinszenierte Passion Jesu - ohne Jesus - durch das akute Leid eines Menschen unterbrochen wird, führt auf der Figurenebene $\mathrm{zu}$ keinerlei Irritationen. Es ist, als setze sich die Ablösung der Protagonisten der Passion von den Ereignissen, die ihnen allererst ihre Identität verleihen, fort in einer vollständigen Trennung der papiernen Passion von leiblichen Passionen, die sich in unmittelbarer Nähe ereignen. 
Die Passion wird darüber hinaus in einer weiteren Hinsicht kupiert. Der theologische Horizont, der sich mit dem Begriff und Ereignis der Passion üblicherweise verbindet, entfällt vollständig: kein «Opfer», keine «Stellvertretung», keine «Sühne». Allein das Kreuz, nicht aber seine traditionellen theologischen Interpretamente sind gegenwärtig. Im Horizont der Figuren (wie er in der Szene ausdrücklich wird), ist das Kreuz keine soteriologische Chiffre, kein Symbol: Es ist ein Kreuz ohne Kreuzigung, ohne Gekreuzigten - und ohne explizite Kreuzesinterpretation. Es ist nichts als ein konkretes Ding, ein materiales Kreuz: zwei Balken, repräsentiert durch entsprechend geformte Papierschnipsel. - Diese zweite Absenz ist bemerkenswert, da sich ja gerade die Passion mit einer ausgeprägten Interpretationspassion verbindet (Passion ebenso zur als auch der Interpretation $\left.{ }^{4}\right)$. Und es handelt sich tatsächlich um eine Absenz: Nicht einmal via negationis, als Ärgernis oder Torheit (1Kor 1,23), kommt eine theologisch konnotierte Bezugnahme ins Spiel. Wenn das Kreuz am Ende zum Fidibus wird, so ist das keine gezielte Profanation des christlichen Zentralsymbols und seiner theologischen Lesarten. Die papierne Passion ist auch dann, was sie immer war: mehr Papier als Passion. Sie kann daher ohne weiteres zum Entzünden einer Zigarre dienen, in deren gemütlichem Qualmen jeder Bedarf nach Passionsinterpretation (der papiernen ebenso wie jener, die vor Augen stehn) erstickt wird. Eingerichtet in einem alles integrierenden "Wat-nich-so-allens-inde-Welt-passiert!»-Horizont, gilt das Interesse dann konsequenterweise einem weiteren Kartoffelpuffer.

Dass Kopelke Wallys mutwillige Auflösung der Szene nur pro forma sanktioniert, Haases Verwandlung der Emmaus-Jünger in Papierbällchen nicht zur Kenntnis nimmt und selbst das Kreuz als Fidibus nutzt, relativiert aber auch eine zweite nahe liegende Vermutung: Werde die Passion schon nicht theologisch qualifiziert, so sei sie doch affirmativ in einen ästhetischen Horizont eingezeichnet. Denn Kopelke nimmt für seine Arbeit ja tatsächlich eine gewisse artistische Kompetenz in Anspruch; ein «janz hibschet Kunststickchen» will er vorgeführt haben, in dem «allens janz jenau» «passt» (34). Diese Bezugnahme ist da, sie ist aber alles andere als emphatisch. Das Kunststückchen kann er, den Diminutiv gleichsam verstärkend, auch als «Spielzeich» (35) bezeichnen. Seine Preisgabe der Passion sowohl als theologisch wie ästhetisch konnotierter Gegenstand reduziert

\footnotetext{
4 Vgl. Ph. Stoellger, Deutung der Passion als Passion der Deutung. Zur Dialektik und Rhetorik der Deutungen des Todes Jesu, in:J. Frey/J. Schröter (Hgg.), Deutungen des Todes Jesu im Neuen Testament, Tübingen 2005, 577-607.
} 
die scheinbare Opposition der parallel angelegten Erzeugung einer gewöhnlichen Mahlzeit und eines Artefakts auf ein Minimum.

\section{IV.}

Aber stimmt das: tatsächlich nur Kreuz, keine Kreuzestheologie und allenfalls eine schwache Kreuzesästhetik? Die dramatische Unterbrechung der ausführlich gezeigten Inszenierung der papiernen Passion lässt leicht vergessen, dass es sich um eine Reinszenierung handelt; die ursprüngliche wird dagegen nur analeptisch erzählt. Auch hier ist nicht ausdrücklich von Kreuzestheologie die Rede, wohl aber von Theologen, die - so wird man annehmen können - streitbare Kreuzestheologen sind. Denn wenn sie ihr Verständnis des Kreuzes für verhöhnt halten, sind sie zur Not auch bereit, ihrer Position durch «eklige Keile» Nachdruck zu verschaffen. Doch erfahren wir weder genau, was ihre diesbezügliche Überzeugung war, noch was ihnen an Kopelkes papierner Passion denn eigentlich missfiel: der Umstand der Passionsdarstellung selbst? dass sie papieren war? der Ort der Darstellung, nämlich «in'ne Kneipe»? der Künstler, der sie gebildet? Wir wissen es nicht. Wir wissen aber, dass die Passion bereits bei ihrer ersten Inszenierung «beinah'» (28) in eine reale Leiderfahrung mündete. Hätte diese Erfahrung Kopelke in besonderer Weise für die subtilen Übergange zwischen Text- und Lebenswelt sensibilisieren können - sie hat es nicht.

Doch ist noch eine zweite Spur erkennbar, welche die Behauptung einer vollständigen Absenz ausdrücklicher Kreuzesinterpretation in Frage stellt. Allerdings ist «ausdrücklich» hier noch schwächer zu verstehen als im Falle der impliziten Kreuzestheologie, welche die rabies theologorum erahnen lässt. Denkbar scheint nämlich, dass Haase, der Statist der Szene, der von Kopelke immer wieder ausdrücklich als Zuschauer seines Kunststücks adressiert wird, nicht nur von diesem beeindruckt ist, sondern eben jenen Transfer leistet, zu dem die Anderen nicht fähig sind. Bei genauerer Betrachtung fällt auf, dass er - als Akteur, der selbst zur Sprache kommt - in einem entscheidenden Moment verstummt: eben dann, wenn die Lücke in der papiernen Passion geschlossen wird durch eine lebensweltliche. Wenn er «Um Gottes-wil-len [...]» (32) ruft, am ganzen Leib zittert und kaum die Papierschnitzel in die Hände bekommt, bleibt offen, was für seinen Zustand eigentlich verantwortlich ist: die blossen Schreie aus dem Hof (kaum ein einmaliges Ereignis) oder ihr Einbruch just in die papierne Passion, deren Skandal in keiner Weise wahr 
genommen wird. Seine letzte Erwähnung im Nebentext - «Herr Haase dreht stumpfsinnig die beiden Jünger von Emmaus zwischen den Fingern» (35) - schafft keine Klarheit. Er erscheint selbst als ein einsamer Emmaus-Jünger, der nach einem Passionstrauma allein gelassen wird, ohne Osterperspektive. Retrospektiv betrachtet, wirft dies ein anderes Licht auf Haases erste Reaktion betreffend Kopelkes Fertigkeit. Seine Antwort auf dessen schlecht verhohlenes Buhlen um Zustimmung - «Nicht doch, Kind! Det interessiert ja den jungen Herrn nich!» - liest sich so nicht länger als willfährige Höflichkeit, sondern als existenziell bestimmtes Wort: «Das Leiden Christi?! Aus Papier?! O gewiss! Sehr! Sehr sogar!» (28) Haases Schweigen, wie jegliches Schweigen notorisch interpretationsoffen, erlaubt zumindest auch dieses Verständnis.

Aufmerksamkeit verdienen schliesslich die selbstbezüglichen Momente, die der Text mit sich führt. - Das erste: Die papierne Passion ist selbst einer Passion ausgesetzt, und dies gleich doppelt, wobei die beiden Leiden sich in entscheidender Weise von einander unterscheiden. Die erste Passion der Passion verdankt sich einer natürlichen Ursache von Aussen, einem Windstoss; dass er ins Zimmer gelangt, ist aber eine Folge der Passion im Hof. Deutlicher als durch diese Verbindung liesse sich die geteilte Ohnmacht der streng isolierten Passionen kaum zeigen. Die zweite Passion, welche die Identität ihrer Elemente schliesslich aufhebt, beruht dagegen auf einem gezielten Akt der Zerstörung von Innen. Und wiederum ist es ein (Windstoss), der klar macht, dass eben gar nicht «allens janz jenau» passt (34). Die (Jesus-)Figur, die zunächst eindringlich um die Herstellung der Szene bat - wie nahe gelegt wird, weniger aus Interesse an ihr, als an einem Kompliment für den Ersatzvater Kopelke -, zerstört sie durch ein Pusten, da die Aufmerksamkeit allzu lange auf ihr ruht, der papiernen Passion und nicht auf ihr, Wally. Von einem erbaulichen und kontrollierten Auf- und Abbau einer Krippenszenerie, die man in der Weihnachtszeit eher erwarten dürfte, ist man hier weit entfernt. Das weihnachtlich-evangelische Motiv der Schwangerschaft wird vielmehr in drastischer Weise verschoben - es erscheint als realistisch verschärfendes Element der Passion im Hof. ${ }^{5}$

\footnotetext{
5 Dass die Passion mehr Papier ist als theologisch hoch konnotierte Szene zeigt sich - vielleicht etwas überdeutlich - auch am konkreten Papier, das zu seiner Herstellung Verwendung findet: Die papierne Passion (verarbeitet) die kleineren und grösseren Passionsgeschichten des «Localanzeigers». Aber auch das führt in der Szene zu keinerlei Bemerkungen.
} 
Das zweite: Was immer mit der papiernen Passion innerhalb der Szene geschieht (im Handlungsverlauf, aber auch im Verlauf seiner Interpretation), jedes Moment lässt sich auch auf Die papierne Passion beziehen. Der Artefakt der papiernen Passion und der papierne Artefakt der Papiernen Passion affizieren sich wechselseitig. Dieses selbstbezügliche Element hat unter Umständen Konsequenzen für die Wahrnehmung des Textes. Durch die Identität des Namens und des Mediums scheint er sich nicht nur irgendwie selbst auszulegen, sondern äusserst selbstkritisch. Der literarische Text gerät unweigerlich in die unvorteilhafte Position des inszenierten heiligen Textes: ein Kunststückchen, das ohnmächtig isoliert von der Lebenswelt steht und schliesslich ganz von ihr absorbiert wird. ${ }^{6}$ Für theologische Leser birgt die Selbstreflexivität des Textes darüber hinaus aber eine besondere Pointe. Denn wie liest der Theologe Die papierne Passion angesichts des Umstands, dass er in ihr repräsentiert wird durch Kollegen, welche die papierne Passion in sehr bestimmter Weise «lesen`? Schliesst er sich ihnen an? Interpretatorische «Keile» für eine unzulässige Passionsdarstellung?

V.

Als Literaturwissenschaftler und Theologe schliesst man sich ihnen eher nicht an. Gerade in der Koppelung beider Perspektiven erweist sich Die papierne Passion als bemerkenswerte Passionsgeschichte, bemerkenswert, weil raffiniert und schwierig. Denn soll die Interpretation tatsächlich ihr gelten (und nicht ihren poetologischen oder literaturgeschichtlichen Kontexten, ihren Autoren, ihrer Rezeption etc.), so nimmt sie den Interpreten fest bei der Hand. Ihre Machart drängt die Interpretation in hohem Mass zur Wiederholung des Interpretandums, zu Paraphrase und Zitat. Die äusserst detaillierte 〈Objektivität〉 des Primärtextes imponiert sich auch im Sekundärdiskurs. Auf die Schnelle ein textferneres fabula docet $\mathrm{zu}$ formulieren, verbietet sich eben so sehr, wie es schwer fällt. Das aber heisst nicht, dass dies leichter fiele, nachdem dem Text in seiner Eigenart ausgiebig Rechnung getragen wurde. Der Text nimmt den Interpreten bei der Hand, er lässt ihn in seiner natura-

\footnotetext{
6 Entsprechend Whitingers Lesart (Johannes Schlaf, s. Anm. 2, 43): «With their depiction of how Kopelke's art relates to its milieu, Holz and Schlaf direct criticism at a traditionally idealistic spaper passion play that fails to adress existing problems. At the same time they encourage the reader to respond with a similar skepticism to their own 〈Paper Passion Play`.»
} 
listischen Küche aber doch auch allein. Wenn über die gesammelten Beobachtungen am Text und schliesslich über ihn hinaus etwas gesagt werden soll, dann vielleicht das Folgende.

Privilegiert man den Aspekt der Passion so liest sich Die papierne Passion gerade in ihrer relativen Theologielosigkeit als kleine Parabel von der Medienverwiesenheit christlicher Passionserinnerung und -reflexion: Die Passion braucht das Papier. Holz/Schlafs Berliner Studie ist insofern auch eine theologische Studie als sie daran erinnert, dass die christliche Passionsgeschichte in ihren Reinszenierungen gegenwärtig bleibt - in jenen der Evangelien, in literarischen, aber auch in theologischen. Sie zeigt dies aber indem sie zeigt, dass diese in problematischer Weise von dem, was ausserhalb ihres Rahmens liegt und was sie allererst zur Erscheinung bringt, entkoppelt werden können. Die Passion braucht das Papier, so passionsanfällig es - sie - selbst auch sein mag. Auch die theologischen Reinszenierungen der Passion (genannt Christologie oder Soteriologie) brauchen eine Sensibilität für ihre Kontexte, für die text- ebenso wie für die lebensweltlichen. Gelingt ihnen dies, bleibt die Passion nicht papieren und das Kreuz nicht Markierung einer gespenstischen Absenz. Gelingt ihnen dies, wird der Gekreuzigte - der auferweckte Gekreuzigte erkennbar als Zentrum christlicher Lebensdeutung.

- Andreas Mauz ist Assistent am Lehrstuhl für Systematische Theologie, insb. Hermeneutik und Fundamentaltheologie an der Universität Zürich. 